\title{
Finding small displacements of recorded speckle patterns: revisited
}

\author{
Hanson, Steen Grüner; Jakobsen, Michael Linde; Chakrabarti, Maumita; Yura, Harold
}

Published in:

Proceedings of SPIE

Link to article, DOI:

$10.1117 / 12.2195631$

Publication date:

2015

Document Version

Publisher's PDF, also known as Version of record

Link back to DTU Orbit

Citation (APA):

Hanson, S. G., Jakobsen, M. L., Chakrabarti, M., \& Yura, H. (2015). Finding small displacements of recorded speckle patterns: revisited. In F. M. Santoyo (Ed.), Proceedings of SPIE (Vol. 9660). [96601J] SPIE -

International Society for Optical Engineering. Proceedings of SPIE - The International Society for Optical Engineering https://doi.org/10.1117/12.2195631

\section{General rights}

Copyright and moral rights for the publications made accessible in the public portal are retained by the authors and/or other copyright owners and it is a condition of accessing publications that users recognise and abide by the legal requirements associated with these rights.

- Users may download and print one copy of any publication from the public portal for the purpose of private study or research.

- You may not further distribute the material or use it for any profit-making activity or commercial gain

- You may freely distribute the URL identifying the publication in the public portal 


\title{
Finding small displacements of recorded speckle patterns - revisited
}

\author{
Steen G. Hanson ${ }^{*}$, Michael Linde Jakobsen ${ }^{\mathrm{a}}$, Maumita Chakrabarti ${ }^{\mathrm{a}}$ \\ and H. T. Yura ${ }^{\mathrm{b}}$ \\ ${ }^{a}$ DTU Fotonik, Department of Photonics Engineering, Technical University of Denmark \\ Frederiksborgvej 399, 4000 Roskilde, Denmark. ${ }^{\mathrm{b}}$ Electronics and Photonics Laboratory, \\ The Aerospace Corporation, P.O. Box 92957, Los Angeles, California, 90009
}

\begin{abstract}
An analytical expression for the bias effect in digital speckle correlation is derived based on a Gaussian approximation of the spatial pixel size and array extent. The evaluation is carried out having assumed an incident speckle field. The analysis is focused on speckle displacements in the order of one pixel, thus having no speckle decorrelation. Furthermore, sensitivity is a main issue wherefore we need speckles close to the pixel size, which means that speckle averaging becomes important, and that Nyquist's criteria may not be fulfilled. Based on these observations, a new correlation method is introduced, which alleviates the need to know the expected shape of the crosscovariance between the original and the off-set recorded speckle pattern. This concept calls for correlating the crosscovariance with the auto covariance, which essentially carries information on the expected shape of the crosscovariance.
\end{abstract}

Keywords: Digital correlation, speckle

\section{Introduction}

The introduction of the laser as a coherent source revealed an interesting feature, namely the destructive and constructive interference of the field scattered off a rough surface. This phenomenon was soon explained, and was termed "speckles" due to its strong modulation that usually approached unity. Further, it was realized that the scattered field was closely connected to the scattering surface, and as such carried valuable information on the dynamics of this surface. A large suite of application of this has later been given, and the theory behind this was explained ${ }^{1}$.

The recording of the speckle pattern was initially performed with photographic techniques strongly limiting the applications. When the Charge Coupled Detector arrays and later the Complementary Metal-Oxide-Semiconductor array (CMOS) appeared, the use of digital correlation really took off, specifically aimed at sub-pixel determination. A method presenting an analysis of four algorithms which were able to register images with subpixel accuracy were published $^{2}$. The four methods that were analyzed were correlation interpolation, intensity interpolation, differential method, and phase correlation. An iterative approach based on intensity correlation was used, and accuracy between 0.01 and 0.05 pixels was claimed. Later Sjödahl gave a theoretical analysis of the sensitivity for speckle displacement for a setup with a given decorrelation, speckle size and pixel size ${ }^{3}$. The standard deviation was proportional to the square of the speckle size and highly relying on the noise. Simultaneously Chen et al. derived subpixel accuracy by using biparabolic fitting near the signal peak ${ }^{4}$. A seminal paper by Sjödahl expanded the analysis of the sensitivity to include phase-only filters and white-light interferometry. Zhou et al. studied an iterative, spatial-gradient-based algorithm, which required the calculation of only first-order spatial derivatives of the images and thus reduced computation complexity ${ }^{5}$. Simulated images with known deformation fields were used to verify their algorithm, as well as to study the impact of speckle size on the accuracy. Based on these simulations, Digital image/speckle correlation was shown to be capable of measuring subpixel displacement greater than 0.005 pixels. Foroosh et al. derived analytic expressions which demonstrated how the phase correlation method can be used to estimate subpixel shifts. The advantages of the method were a high accuracy and providing closed-form expressions for the error ${ }^{6}$. This method addressed incoherent imagery.

SPECKLE 2015: VI International Conference on Speckle Metrology, edited by Fernando Mendoza Santoyo, Eugenio R. Méndez, Proc. of SPIE Vol. 9660, 96601J · (c) 2015 SPIE · CCC code:

$0277-786 \mathrm{X} / 15 / \$ 18 \cdot$ doi: $10.1117 / 12.2195631$ 
The combination of high accuracy for digital correlation and industrial applications has been large in the last decade. Two interesting and challenging approaches using subpixel estimation are worth mentioning, viz. a setup for measuring expansion of humid paper ${ }^{7}$ and flow measurement by Particle Imaging Velocimetry (PIV) ${ }^{8}$.

Two recent papers have been addressing the issue of increasing the accuracy in digital speckle correlation. Wang et al. matched the positions of singularities in the two recordings, and found an accuracy of 0.015 pixel by tracking the motion of the singularities. Their method were used with ordinary incoherent images and involved time-consuming computation ${ }^{9}$. Meng et al. ${ }^{10}$ used an iterative and spatial-gradient algorithm to derive sub-pixel accuracy whereas Guizar used nonlinear optimization to address the same issue ${ }^{11}$.

In this paper we will address two issues. Firstly we will display an analytic method with which we can provide analytic expressions for the bias effect - sometimes named "systematic error"- associated with the measurement of speckle displacement. Secondly, realizing that we do not always know the expected shape of the crosscovariance between two recording, which is beneficial for the peak-finding procedure, we introduce an alternative method. This method relies on using a quadratic peak-finding algorithm to the correlation between averaged autocorrelation and the measured crosscovariance. The rationale behind this is that the crosscovariance is an off-set version of the auto-covariance. It will here be argued that this method facilitates a reduction of the bias effect usually associated with a quadratic fitting procedure.

\section{Theoretical evaluation of bias effects.}

The image array will here be treated as a $1 \mathrm{D}$ linear array. The expansion into a $2 \mathrm{D}$ array is trivial and will not be given here. In order to arrive at a useful and general expression for the expected bias effects - sometimes named "systematic error" - we will assume each pixel to be Gaussian apodized with a width $\omega_{p}$ (1/e intensity halfwidth) and an overall Gaussian apodized width $\omega_{0}$ (1/e intensity halfwidth). This means that the effective number of pixels is $\omega_{0} / \omega_{p}$.

The irradiance incident on the linear array stretching along the $x$-axis is $I[x]$ and is assumed to be independent of the orthogonal axis in the image plane. The response from pixel number $j$ thus becomes:

$$
S_{1}(j)=\int_{-\infty}^{\infty} I[x] \exp \left[-\frac{(x-j \Lambda)^{2}}{\omega_{p}^{2}}\right] \exp \left[-\frac{x^{2}}{\omega_{0}^{2}}\right] d x,
$$

Where the pitch of the linear array is given by $\Lambda$. The ratio of $\omega_{p}$ to $\Lambda$ determines the effective fill factor for the pixels. In this way a small fill factor corresponds to a delta-sampling of the irradiance, while a fill factor close to unity gives an integration of the incident irradiance including some spill-over from one pixel to an adjacent pixel, due to the Gaussian sensitivity assumed for the pixels.

The response from pixel number $j$ ' after shifting the irradiance a distance of $\Delta x$ is

$$
S_{2}\left(j^{\prime}\right)=\int_{-\infty}^{\infty} I_{2}[x-\Delta x] \exp \left[-\frac{\left(x-j^{\prime} \Lambda\right)^{2}}{\omega_{p}^{2}}\right] \exp \left[-\frac{x^{2}}{\omega_{0}^{2}}\right] d x,
$$

The crosscorrelation $C[\Delta j]$ between the two recordings, one before the displacement and one after the shift becomes:

$$
C_{\text {pixel }}[\Delta j]=\sum_{j=-\infty}^{\infty} S_{1}[j] S_{2}[j-\Delta j]
$$

Needless to say, the crosscorrelation is given for integer values of $\Delta j$ only. But the aim is to derive the value of $\Delta x$ with an accuracy given by fractions of the pixel pitch $\Lambda$. As previously stated, we will seek an analytical expression for any bias effect that may arise, specifically in the case of the speckle size not being much larger than the pixel size, and for 
speckle displacements of the order of the pixel pitch. On the other hand, we will assume no decorrelation of the speckle pattern as it is shifted between the recordings. The "noise" that obstructs a precise determination of the speckle displacement is given by the sampling and the associated integration of the speckle pattern.

Next, we introduce the autocorrelation of the speckle patterns before and after the displacement. If the speckle pattern arises from scattering of a Gaussian beam off a rough surface, and the speckle pattern is established after free space propagation, the intensity correlation will attain a Gaussian form:

$$
\left\langle I\left[x_{1}\right] I\left[x_{2}\right]\right\rangle=\left\langle I\left[x_{1}\right]\right\rangle\left\langle I\left[x_{2}\right]\right\rangle\left(1+\exp \left[-\frac{\left(x_{1}-x_{2}\right)^{2}}{\rho_{0}^{2}}\right]\right)
$$

where $\rho_{0}$ is the radius of the average speckle and angular brackets indicate "ensemble average." The auto-covariance $A[\Delta x]$, which contains the space-dependent contribution is given by:

$$
A[\Delta x] \equiv\left\langle I_{1}[x] I_{1}[x-\Delta x]\right\rangle-\left\langle I_{1}[x]\right\rangle\left\langle I_{1}[x-\Delta x]\right\rangle \propto \exp \left[-\frac{\Delta x^{2}}{\rho_{0}^{2}}\right] .
$$

We have here assumed the statistics for the irradiance to be space-independent. Further, having assumed that we have no speckle decorrelation, the crosscovariance between the original and the offset irradiance thus becomes:

$$
\begin{gathered}
C_{\text {irradiance }}[\Delta x] \equiv\left\langle I_{1}[x] I_{2}\left[x-\Delta x-\Delta x_{0}\right]\right\rangle-\left\langle I_{1}[x]\right\rangle\left\langle I_{2}[x-\Delta x]\right\rangle= \\
\left\langle I[x] I\left[x-\Delta x-\Delta x_{0}\right]\right\rangle-\langle I[x]\rangle\left\langle I\left[x-\Delta x-\Delta x_{0}\right]\right\rangle \propto \exp \left[-\frac{\left(\Delta x-\Delta x_{0}\right)^{2}}{\rho_{0}^{2}}\right],
\end{gathered}
$$

where the induced shift of the irradiance is $\Delta x_{0}$. We can now derive the ensemble average of the crosscovariance of the pixel values by inserting Eq.5 into the expressions for the pixel values before and after displacement (Eqs.1 and 2) and use the expression for the irradiance covariance, Eq.6:

$$
C_{\text {pixel }}[\Delta j]=\sum_{j=-\infty}^{\infty} \int_{-\infty}^{\infty} \int_{-\infty}^{\infty}\left\langle I_{1}[x] I\left[x_{2}\right]\right\rangle \exp \left[-\frac{\left(x_{1}-j \Lambda\right)^{2}}{\omega_{p}^{2}}\right] \exp \left[-\frac{x_{1}^{2}}{\omega_{0}^{2}}\right] \exp \left[-\frac{\left(x_{2}-(j-\Delta j) \Lambda\right)^{2}}{\omega_{p}^{2}}\right] \exp \left[-\frac{x_{2}^{2}}{\omega_{0}^{2}}\right] d x_{1} d x_{2} .
$$

This integral can be evaluated analytically for the given intensity covariance distribution Eq.[5], and gives the result:

$$
C_{\text {pixel }}[\Delta j]==\exp \left[-\frac{(\Delta j \Lambda)^{2}}{2 \omega_{0}^{2}}\right] \exp \left[-\frac{\left(\Delta x_{0}-\Delta j \Lambda\right)^{2}}{\rho_{0}^{2}+\omega_{p}^{2}}\right] \Theta\left[3,-\frac{\Delta j \pi}{2}, \exp \left[-\frac{\pi \omega_{0}^{2}}{2 \Lambda^{2}}\right]\right],
$$

where $\Theta\left[3,-\frac{\Delta j \pi}{2}, \exp \left[-\frac{\pi \omega_{0}^{2}}{2 \Lambda^{2}}\right]\right]$ is the Elliptic Theta function, which for large number of pixels is a very weak oscillatory function around unity with a period giving a maximum value for $\Delta j=n \frac{4}{\pi}$, where $n$ is an integer. An decrease in the number of pixels will increase the influence of this oscillation. This contribution is thus of major importance for arrays with a small number of pixels. In arriving at the above expression, we have assumed that the width of the array exceeds both the speckle size and the pixel width, i.e. $\omega_{0} \gg\left\{\rho_{0}, \omega_{p}\right\}$. The expression for the pixel covariance in Eq.8 contains two leading exponential contributions, besides the elliptic theta function. The first exponential contribution defines the decay of the covariance as the pixel displacement approaches the array edge and is 
therefore of minor importance for the scene we are investigating. The second exponential gives the main contribution to the shape and position of the covariance. It is noted that the peak position for the covariance doesn't fall exactly at $\Delta j=\Delta x_{0} / \Lambda$, which usually is not an integer value. Therefore, interpolation in the vicinity of the peak has to be performed. Furthermore, the expression depends on two variables, namely the number of pixels given by $\omega_{0} / \Lambda$ and the geometric sum of the pixel size and the speckle size i.e. $\sqrt{\rho_{0}^{2}+\omega_{p}^{2}}$.

The case of major interest for us is the case, where we encounter speckle shifts below one pixel. Besides, we aim at acquiring measurements at a high accuracy, which calls for a speckle sizes close to the period of the pixels in the array. The question arises naturally whether - or to what extent - we will encounter bias effects? Therefore we will only use the pixel values for displacement $\left\{[-1,0,1\}\right.$ for $C_{\text {pixel }}[\Delta j]$. These values can be found analytically by using the three displacement values in Eq.[8]. A quadratic approximation to these three values gives the interpolated average peak position placed at the fractional pixel number:

$$
\Delta j_{\text {peak }}=\frac{C_{\text {pixel }}[-1]-C_{\text {pixel }}[1]}{2\left(C_{\text {pixel }}[1]+C_{\text {pixel }}[-1]-2 C_{\text {pixel }}[0]\right)} .
$$

Combining this expression with the average values of the covariance, Eq.[8], provides an analytical expression and therefore valuable insight into the bias effect one can expect for given values of the pixel size, the pitch and length of the array and the speckle size. Some revealing examples will here be given.

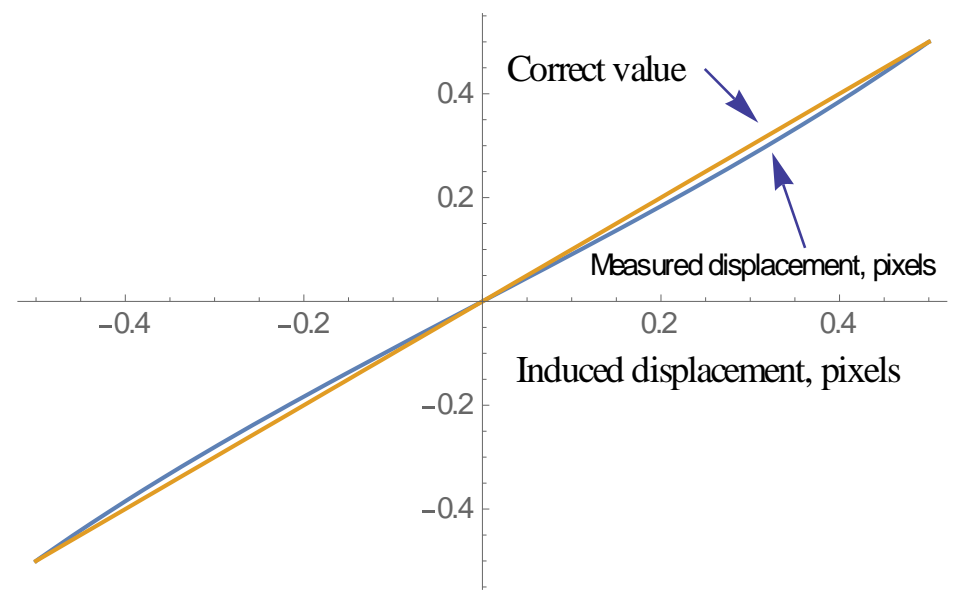

Fig.1. Bias effect incurred for the values: $\Lambda=1, \rho_{0}=2, \omega_{0}=100$ and $\omega_{p}=0.7$.

In the following, we will focus on the difference between the induced displacement $\Delta x_{0}$ and the measured displacement $\Delta j_{0}$. The difference is the bias effect, here $\Delta j_{0}-\Delta x_{0}$ and its dependence on the free parameters $\Lambda, \rho_{0}, \omega_{0}$ and $\omega_{p}$ will be investigated below. In the following we will tacitly assume that the detector pitch is $\Lambda=1$.

Fig. 1 shows the correct and the measured average measured shift as a function of the induced speckle shift, here given for values between -.5 and .5. If the induced displacement exceeds 0.5 , the covariance values used for estimating the peak position will be shifted in order to have reading on each side of the maximum covariance value. In this case the bias effect will be repeated, as shown in Fig. 2. 


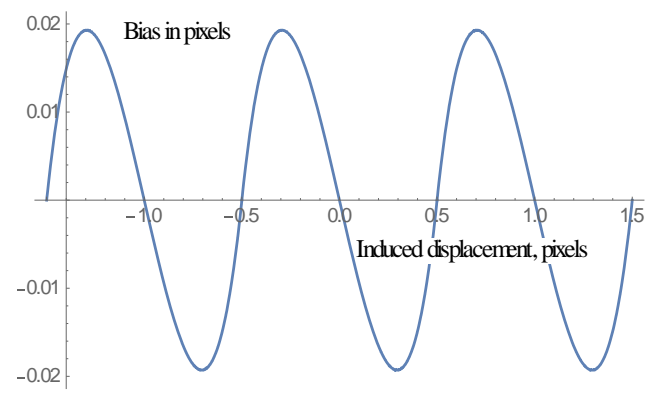

Fig. 2. Bias effect measured in pixels for the parameters: $\Lambda=1, \rho_{0}=2, \omega_{0}=1000$ and $\omega_{p}=0.7$.

It is noted that the bias tends to "pull" the measured value towards the closest integer pixel value, e.g. for the induced displacement lying between zero and one half of the pitch length, the measured value will be slightly closer to zero, i.e. a negative bias. For a displacement of one half of a pixel, there is no bias effect, and when the displacement lies between one half and unity, the bias is positive, i.e. being pulled towards unity.

In the above example, the bias effect is in worst case close to $2 \%$. Fig 3 shows - for a given setup - how the bias effect depends on the speckle size. The bias effect is shown to decrease as the speckle size increases and thus the Nyquist criteria is obeyed.

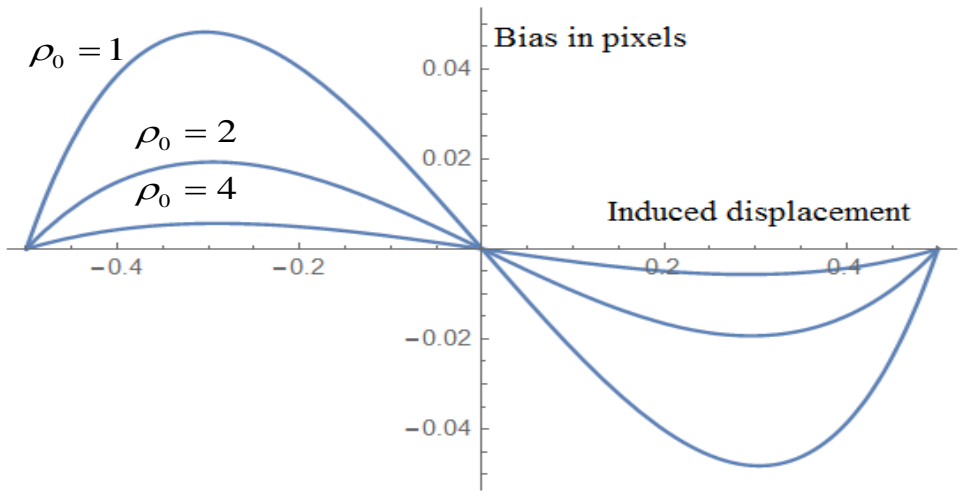

Fig.3. The bias effect for varying the induced displacement from -.5 to .5 pixels for three values of the speckle size $\rho_{0}$. The array

$$
\text { parameters are } \Lambda=1, \omega_{0}=1000 \text { and } \omega_{p}=0.7 \text {. }
$$

Finally, Fig. 4 shows how the bias effect is increased when the pixel size - and thus the fill factor- is reduced, and the sampling approaches a point sampling without the inherent low-pass filtering that occurs when the pixel size approaches the pitch of the array. 


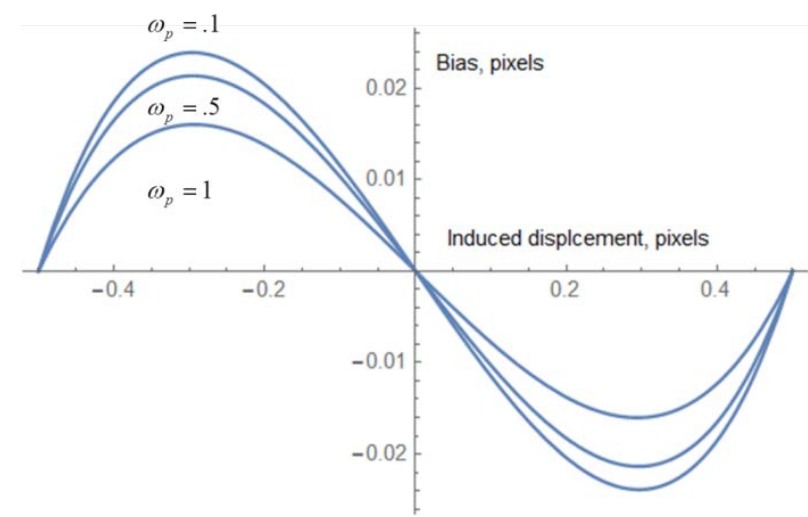

Fig.4. Bias effect as a function of the pixel size, i.e. the fill factor for the array. The parameters are: $\Lambda=1, \rho_{0}=2, \omega_{0}=1000$.

Finally, the bias effect measured in pixels as a function of the speckle size is given in Fig.5 for various values of the pixel width, where the pixel pitch is unity.

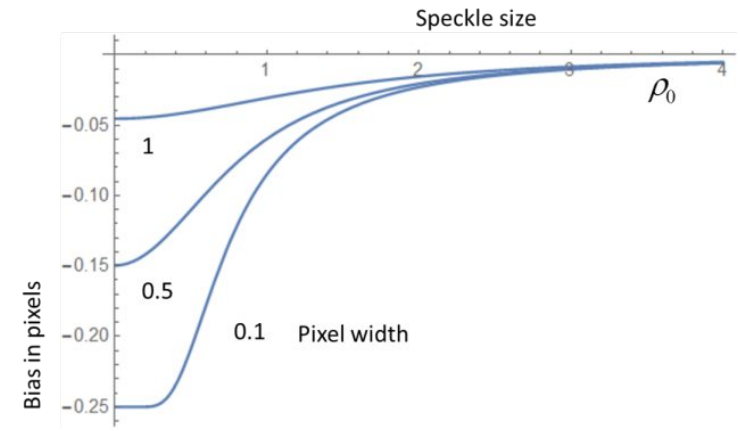

Fig.5. Bias effect measured in pixels as a function of the speckle size. The width of the detector array is $\omega_{0}=1000$.

It has to be mentioned that the bias effect will be close to negligible had we chosen a Gaussian fit to the crosscovariance if the number of pixels was large.

\section{Peak finding}

Next, we will introduce a method, which we will argue will reduce the bias effect when a quadratic approximation is used for peak finding. The rationale for this is that a better approximation than the quadratic has to be found, provided that we do not know the exact shape of the crosscovariance $C_{\text {pixel }}[\Delta j]$. It's evident that a good estimate of this shape is given by the autocovariance, making it plausible that correlating the measured crosscovariance with the auto-covariance of one of the recordings will give a better estimate of the displacement of the pattern that was incident on the detector array. The drawback of this will be an increase in the width of this function, here named the "second order covariance," resulting in a possible slight decrease in the accuracy. The second order covariance is given by:

$$
C_{2}[\Delta j]=\left(\sum_{j=-\infty}^{\infty} S_{1}[j] S_{2}[j-\Delta j]\right) \otimes\left(\sum_{j=-\infty}^{\infty} S_{1}[j] S_{1}[j-\Delta j]\right)
$$

where $\otimes$ stands for “correlation”. 
Before giving an example of the benefit of this approach, a short discussion of the physical meaning in the spectral regime will be given. The auto- and crosscovariance equals the inverse Fourier transform of the product of each of the Fourier transform of the incoming signals, where one of the transforms is complex conjugated. Using this, we get

$$
C_{2}\left[\Delta x_{2}\right]=\mathfrak{I}^{-1}\left[\mathfrak{I}\left[\mathfrak{I}^{-1}\left[\mathfrak{I}\left[s_{1}\right] \mathfrak{I}^{*}\left[s_{2}\right]\right] \mathfrak{I}^{*}\left[\mathfrak{I}^{-1}\left[\mathfrak{I}\left[s_{1}\right] \mathfrak{I}^{*}\left[s_{1}\right]\right]\right]\right]\right],
$$

where $\mathfrak{I}$ and $\mathfrak{I}^{-1}$ stands for Fourier transform and inverse Fourier transform, respectively.

It seems a little complicated, but a closer look reveals it’s rather simple. Next, this is reduced to give:

$$
\begin{gathered}
C_{2}[\Delta j]=\mathfrak{I}^{-1}\left[\mathfrak{I}\left[s_{1}\right] \mathfrak{J}^{*}\left[s_{2}\right] \mathfrak{J}^{*}\left[s_{1}\right] \mathfrak{I}\left[s_{1}\right]\right] \\
\text { or } \\
C_{2}[\Delta j]=\mathfrak{J}^{-1}\left[\mathfrak{J}\left[s_{1}\right] \mathfrak{J}^{*}\left[s_{2}\right]\left|\mathfrak{I}\left[s_{1}\right]\right|^{2}\right]=\mathfrak{I}^{-1}\left[\mathfrak{I}\left[s_{1}\right] \mathfrak{I}^{*}\left[s_{2}\right] P_{1}[\omega)\right],
\end{gathered}
$$

where $\left.P_{1}(\omega) \equiv \mid \mathfrak{I}\left[s_{1}\right]\right]^{2}$ is the power spectrum of one of the ingoing signals. This shows that the cross spectrum is merely weighted by the spectrum for the first signal and then inversely Fourier transformed in order to arrive at the new covariance, from which a better estimate of the peak position is assumed to be found. A second way to interpret the above result is to realize that the spectral content of the two signals' contribution to the cross covariance is weighted by the amount of power in one of the signals - here signal number one is arbitrarily used. If we assumed that $\left|\mathfrak{J}\left[s_{1}\right]\right|^{2}=1$, i.e. an equal contribution for all spectral components, we would arrive at the original crosscovariance, i.e. $C_{2}[\Delta j]=C_{\text {pixel }}[\Delta j]$. In the other extreme, where the signal is highly band-limited, the cross-spectrum of the two signals that has to be correlated will be spectrally truncated and the noise reduced.

In case a series of measurements are to be performed with the same optical setup in order to derive a set of displacements, it might be beneficial to use an averaged value of the power spectrum $P(\omega)$ in Eq.13 in order to reduce the influence of any noise in the auto covariance.

Table 1. Combinations of induced and measured speckle shift for three values of the displacement

\begin{tabular}{|c|c|c|c|c|}
\hline Array pitch/speckle size & Induced Shift & Method & Measured shift & Variance \\
\hline & pixels & & pixels & pixels \\
\hline 0.4 & -2.2 & $C_{2}$ & 2.312 & 0.014 \\
\hline 0.4 & -2.8 & $C_{2}$ & -2.690 & 0.020 \\
\hline 0.2 & -2.2 & $C_{2}$ & -2.231 & 0.009 \\
\hline 0.2 & -2.8 & $C_{2}$ & -2.764 & 0.011 \\
\hline 0.2 & -2.5 & $C_{2}$ & -2.500 & 0.012 \\
\hline 0.4 & -2.5 & $C_{2}$ & -2.31 & 0.013 \\
\hline 0.4 & -2.2 & $C_{1}$ & -2.356 & 0.009 \\
\hline 0.4 & -2.8 & $C_{1}$ & -2.637 & 0.006 \\
\hline 0.2 & -2.2 & $C_{1}$ & -2.244 & 0.008 \\
\hline 0.2 & -2.8 & $C_{1}$ & -2.750 & 0.012 \\
\hline 0.2 & -2.5 & $C_{1}$ & -2.500 & 0.006 \\
\hline 0.4 & -2.5 & $C_{1}$ & -2.500 & 0.002 \\
\hline
\end{tabular}


A series of simulations were made by a simulated linear speckle pattern with two speckle sizes, namely 5 and 2.5 speckles per array period. The pixels sampled the speckle pattern at the center of the pixel, thus no integration of the irradiance across the pixel was performed in the simulations, the results of which are summarized in Table 1. For each combination of speckle size and induced displacement of the speckle pattern, 1000 independent realizations were made and the average measured displacement and its variance were calculated. In all cases we used a quadratic fit over 12 pixels for both the ordinary crosscovariance $C_{\text {pixel }}$, Eq.3, and for the above introduced covariance $C_{2}[\Delta j]$, Eq.10.

The result of these measurements provides the following conclusions in accordance with the predictions given by the analytic results arrived at in the previous chapter:

- No bias is observed for displacements close to one half of a pixel

- The direction of the bias effects is in accordance with the analytic result

- The "new" crosscovariance $C_{2}[\Delta j]$ gives less bias effect

- The "new" crosscovariance $C_{2}[\Delta j]$ gives slightly larger variance on the estimation of the peak position.

\section{Conclusion}

First an analytical expression for the bias effect was derived in case a quadratic fit to the crosscovariance was used. The irradiance of the linear detector array was here assumed to be a speckle structure with a given and finite speckle size. The result was obtained by approximating the pixel sensitivity with a spatial Gaussian function. Furthermore, the spatial sensitivity of the detector array was assumed to taper off at the edges according to a Gaussian function. An increase of the bias affect was shown to occur when the speckle size approached the pixel size and when the fill factor for the detector array was decreased.

Next, based on the above, it was established that a quadratic fitting function might not provide the best result, in case we have no knowledge on the expected shape of the crosscovariance. In this case, a good fitting function could be the autocovariance, which merely has the same shape as the covariance, in case we neglect the influence of the digitization.

In both the treated examples, we have addressed the case where the speckles - or the feature size in case of ordinary imagery - have a size comparable with the pixel size. Furthermore, we have assumed no decorrelation of the underlying irradiance during the shift. On the other hand, we have not assumed a large number of detector elements, which opens up for using this approach in cases where the information from a detector array is divided up in smaller sub-areas with a limited number of pixels. And, needless to say, the current approach can easily be expanded to cover the outcome from a 2D detector array.

\section{ACKNOWLEDGMENT}

The work was partially funded by the Danish Council for Technology and Innovation under the Innovation Consortium LICQOP, grant \#2416669. 


\section{REFERENCES}

[1] Yamaguchi, I., "Speckle Displacement and Decorrelation in the Diffraction and Image Fields for Small Object Deformation,” Opt. Acta Int. J. Opt. 28(10), 1359-1376 (1981).

[2] Tian, Q.., Huhns, M. N., “Algorithms for subpixel registration,” Comput. Vision, Graph. Image Process. 35(2), 220-233 (1986).

[3] Sjödahl, M.., Benckert, L. R., "Electronic speckle photography: analysis of an algorithm giving the displacement with subpixel accuracy.,” Appl. Opt. 32(13), 2278-2284 (1993).

[4] Chen, D. J., Chiang, F. P., Tan, Y. S.., Don, H. S., "Digital speckle-displacement measurement using a complex spectrum method.,” Appl. Opt. 32(11), 1839-1849 (1993).

[5] Zhou, P.., Goodson, K. E., "Subpixel displacement and deformation gradient measurement using digital image/speckle correlation (DISC),” Opt. Eng. 40(8), 1613 (2001).

[6] Foroosh, H., Zerubia, J. B.., Berthod, M., “Extension of phase correlation to subpixel registration,” IEEE Trans. Image Process. 11(3), 188-199 (2002).

[7] Larsson, L., Sjödahl, M.., Thuvander, F., "Microscopic 3-D displacement field measurements using digital speckle photography,” Opt. Lasers Eng. 41(5), 767-777 (2004).

[8] Roesgen, T., “Optimal subpixel interpolation in particle image velocimetry,” Exp. Fluids 35(3), 252-256 (2003).

[9] Takeda, M., Wang, W., Hanson, S. G.., Miyamoto, Y., “Optical vortex metrology.”

[10] Meng, L. B., Jin, G. C.., Yao, X. F., “Application of iteration and finite element smoothing technique for displacement and strain measurement of digital speckle correlation,” Opt. Lasers Eng. 45(1), 57-63 (2007).

[11] Guizar-Sicairos, M., Thurman, S. T.., Fienup, J. R., "Efficient subpixel image registration algorithms.,” Opt. Lett. 33(2), 156-158 (2008). 